\title{
Video Article \\ Confocal Microscope-Based Laser Ablation and Regeneration Assay in Zebrafish Interneuromast Cells
}

\author{
Bryan A. Volpe ${ }^{1}$, Teresa H. Fotino ${ }^{1}$, Aaron B. Steiner ${ }^{1}$ \\ ${ }^{1}$ Department of Biology, Pace University \\ Correspondence to: Aaron B. Steiner at asteiner@pace.edu \\ URL: https://www.jove.com/video/60966 \\ DOI: doi:10.3791/60966
}

Keywords: Developmental Biology, Issue 159, lateral line, interneuromast, neuromast, hair cell, mantle cell, supporting cell, ET20, regeneration, laser ablation, confocal, $405 \mathrm{~nm}$

Date Published: 5/20/2020

Citation: Volpe, B.A., Fotino, T.H., Steiner, A.B. Confocal Microscope-Based Laser Ablation and Regeneration Assay in Zebrafish Interneuromast Cells. J. Vis. Exp. (159), e60966, doi:10.3791/60966 (2020).

\section{Abstract}

Hair cells are mechanosensory cells that mediate the sense of hearing. These cells do not regenerate after damage in humans, but they are naturally replenished in non-mammalian vertebrates such as zebrafish. The zebrafish lateral line system is a useful model for characterizing sensory hair cell regeneration. The lateral line is comprised of hair cell-containing organs called neuromasts, which are linked together by a string of interneuromast cells (INMCs). INMCs act as progenitor cells that give rise to new neuromasts during development. INMCs can repair gaps in the lateral line system created by cell death. A method is described here for selective INMC ablation using a conventional laserscanning confocal microscope and transgenic fish that express green fluorescent protein in INMCs. Time-lapse microscopy is then used to monitor INMC regeneration and determine the rate of gap closure. This represents an accessible protocol for cell ablation that does not require specialized equipment, such as a high-powered pulsed ultraviolet laser. The ablation protocol may serve broader interests, as it could be useful for the ablation of additional cell types, employing a tool set that is already available to many users. This technique will further enable the characterization of INMC regeneration under different conditions and from different genetic backgrounds, which will advance the understanding of sensory progenitor cell regeneration.

\section{Introduction}

At the core of most progressive hearing loss lies the destruction of sensory hair cells, which transduce extrinsic auditory stimuli into nerve impulses detectable by the brain. Cochlear hair cell death causes permanent hearing loss, as adult mammals lack the ability to regenerate these cells following damage. Conversely, non-mammalian vertebrates such as zebrafish can regenerate hair cells lost to acoustic trauma or ototoxic insult. The zebrafish mechanosensory lateral line is a simple and easily manipulated organ system that can be used to study hair cell regeneration ${ }^{1,2,3}$

The lateral line consists of small sensory patches called neuromasts, which are connected during development by a string of elongated cells known as interneuromast cells (INMCs). Given their proliferative capacity as apparent stem cells that give rise to new hair cell-containing organs, the behavior of INMCs is of great interest to the community ${ }^{4,5}$. Much of the research pertaining to INMCs has characterized suppression of their proliferation by nearby Schwann cells that wrap around the lateral line nerve, likely through an inhibitor of Wnt/ $\beta$-catenin signaling. ${ }^{6,7,8}$. While the regulation of INMC proliferation and differentiation into new neuromasts has been well-described, the mechanisms governing INMC regrowth after ablation have not been elucidated. This protocol serves as a means of ablating individual INMCs and analyzing their subsequent regenerative behavior.

A variety of methods for destroying hair cells, supporting cells, and entire neuromasts have been published, along with methodologies for monitoring recovery. Chemical ablation works well for hair cells, but doses of toxic chemicals such as $\mathrm{CuSO}_{4}$ must be significantly increased to remove other lateral line cell types ${ }^{9}$. While electroablation under fluorescence microscopy is an effective and simple means of destroying INMCs to study regeneration, it is difficult to target narrowly and, therefore, is likely to produce significant collateral damage. As a result, this can mask aspects of INMC-specific behaviors ${ }^{10,11}$. Laser ablation protocols have been employed, but they require the use of specialized equipment not necessarily present in the average laboratory or imaging facility (i.e., high-powered pulsed UV lasers ${ }^{12}$ ). The method described here can be performed with any laser-scanning confocal microscope outfitted with the common $405 \mathrm{~nm}$ laser, usually used for imaging DAPI and other blue fluorophores. An advantage of this method is that confocal imaging can be performed immediately before and after cell damage without engaging any additional laser control systems or transferring to another microscope equipped with a pulsed laser.

A similar method has been described for ablating neurons in the zebrafish spinal cord ${ }^{13}$. However, the previous method requires a FRAP module for cell ablation, which is not a requirement for this protocol. Furthermore, given the distinct properties of different cell types (i.e., brightness of transgene fluorescence, depth within the body, and cell shape), it is likely that significant modifications will be needed depending on the cell type used. The protocol detailed here is more likely to be effective for more superficial cells, as supported by a demonstration of sensory hair cell ablation using the same method. Also outlined is a regeneration assay to assess INMC recovery rates after ablation, which was not a feature of the aforementioned study. 
The laser-based cell ablation protocol detailed herein captures the regenerative capacity of INMCs through the optimization of laser conditions, pre- and post-ablation imaging, and time-lapse microscopy. These elements come together to comprehensively portray INMC regrowth and gap closure in its entirety. While this protocol is used here to destroy INMCs, it may be applied to other work that requires reliable and effective assessment of cellular ablation. It is also an accessible technology, since it can be conducted on any confocal microscope equipped with a 405 $\mathrm{nm}$ laser.

\section{Protocol}

All animal work was approved by the Pace University Institutional Animal Care and Use Committee under protocol 2018-1.

\section{Preparation of zebrafish larvae}

1. Set up mating 3-5 days prior to conducting the experiment, such that larvae will be 2 days post-fertilization (dpf) to 4 dpf in age when mounted for laser ablation.

NOTE: The Et(krt4:EGFP)sqEt20 (abbreviated as ET20) enhancer trap line, in which interneuromast cells are clearly labeled with enhanced green fluorescent protein (eGFP), allows for relatively easy cell identification and ablation ${ }^{11,14}$. For confirmation of laser ablation specificity and flexibility, ET20 fish are bred with $T g$ (neuroD:tdTomato) fish, in which the lateral line nerve is labeled with red fluorescent protein or with Tg(myo6b:ßactin-GFP) to label sensory hair cells with GFP ${ }^{15,16}$.

2. After embryo collection the following day, incubate embryos at $28.5^{\circ} \mathrm{C}$ in E3 medium containing $0.0001 \%$ methylene blue until ready to anesthetize and mount.

\section{Preparation of low gelling agarose and tricaine solutions}

1. To a $250 \mathrm{~mL}$ glass Erlenmeyer flask, add $48 \mathrm{~mL}$ of $1 \times$ E 3 medium, $2 \mathrm{~mL}$ of $15 \mathrm{mM}$ tricaine stock solution, and $0.5 \mathrm{~g}$ of low-melting agarose.

2. Microwave the solution on high for $30 \mathrm{~s}$ and swirl (wearing heat-protective gloves) to dissolve the agarose. Repeat the $30 \mathrm{~s}$ heating cycles until agarose is fully dissolved.

3. Store the agarose in an incubator heated to $42{ }^{\circ} \mathrm{C}$ until ready to use.

\section{Anesthetization of zebrafish larvae}

1. To a $50 \mathrm{~mL}$ conical tube, add $24 \mathrm{~mL}$ of $1 \times \mathrm{x} 3$ medium and $1 \mathrm{~mL}$ of tricaine stock solution $(15 \mathrm{mM})$ to a final concentration of $600 \mu \mathrm{M}$. Swirl to mix.

2. Load one of the six wells of a flat-bottomed cell culture plate with $8 \mathrm{~mL}$ of $\mathrm{E} 3$ containing $600 \mu \mathrm{M}$ tricaine.

3. Use a transfer pipette to move zebrafish larvae into a $74 \mu \mathrm{m}$ mesh-bottomed transfer insert.

4. Transfer the mesh-bottomed insert into the well of the six-well plate containing the E3 + tricaine solution. Allow the larvae to remain in the well for a minimum of $3 \mathrm{~min}$ or until fully anesthetized. Test anesthesia by tapping the insert. Larvae should not startle and swim in response to the tap.

\section{Fluorescent screening of anesthetized zebrafish larvae}

1. Pipette one anesthetized larva per well onto 12 well hydrophobic-coated slides for screening. Ensure that there is minimal curvature of the meniscus formed in each well of the slide to reduce optical distortion when screening. This can be done by removing a small volume of E3 from each well after placing the larvae.

2. Under a 10x objective on an upright microscope (equipped with a mercury arc lamp, metal-halide lamp, or LED light source and an appropriate filter cube), screen for larvae that express eGFP in the neuromasts and interneuromast cells of the lateral line system.

1. Select larvae with stronger expression resulting in brighter eGFP, which presumably are homozygous for the transgene. NOTE: In the ET20 line, eGFP is expressed in a ring of mantle cells surrounding each neuromast as well as the narrow strip of interneuromast cells connecting these organs. Stronger eGFP expression (brighter fluorescence) contributes to more effective ablation.

2. To examine the specificity of ablation, use double-transgenic larvae with both the ET20 and Tg(neuroD:tdTomato) transgenes. If screening for $\operatorname{Tg}$ (neuroD:tdTomato) carriers, use the RFP filter cube set to identify a bright red patch just posterior to the ear representing the posterior lateral line ganglion.

3. To ablate hair cells in the lateral line neuromasts, use the Tg(myo6b:ßactin-GFP) transgenic line and screen for GFP localization in the center of each neuromast.

3. Select larvae with stereotypical spacing of the neuromasts. Differences in the spacing between neuromasts may indicate abnormal neuromast deposition during development, suggesting defective migratory behavior or cell proliferation in the lateral line primordium ${ }^{17}$. Such defects may also affect migratory or regenerative behavior of the INMCs following ablation.

NOTE: Common defects in spacing include an unusually large distance between the anterior-most neuromast deposited by the first migrating primordium (prim1L1) and the second neuromast deposited by the same primordium (prim1L2), often associated with crowding of the more posterior neuromasts.

\section{Mounting larvae for laser ablation and imaging}

1. Pipette 3 - 4 anesthetized larvae into a small droplet of E3/tricaine solution in the center of a cover slip-bottomed dish (35 mm dish with a 14 $\mathrm{mm}$ number 1.5 coverslip). Remove excess solution so that the larvae remain in a small droplet, just large enough to contain them.

2. Transfer the dish to the stage of a binocular stereo microscope and manipulate the zoom and focus so that all larvae are in the field of view. 
3. Remove the Erlenmeyer flask containing the low melting point agarose from the heated incubator. Use a transfer pipette to transfer agarose solution onto the cover slide to produce a thin layer. Draw off excess agarose until the liquid just fills the well at the bottom of the dish, taking care not to aspirate any larvae.

4. Quickly arrange the larvae in the agarose solution using a hair knife or hair loop so that they are aligned with each other and oriented with rostral to the left.

5. Gently press the larvae down against the glass with the hair knife, such that they lie in profile with their right sides down. Larvae older than $3 \mathrm{dpf}$ tend to float due to their inflated swim bladders, so they may need to be repeatedly pressed down against the glass until the agarose begins to gel.

6. After about $60 \mathrm{~s}$ the agarose will start to solidify, and the larvae will not be able to be reoriented. Allow approximately 5 minutes at room temperature for the agarose on the cover slip to completely solidify. Once the agarose has solidified, use a transfer pipette to fill the dish halfway with E3 containing 1x tricaine.

\section{Locating prospective targets and pre-ablation imaging}

1. Turn on the power to the laser-scanning confocal microscopy system and initialize through the integrated imaging software. Select the $63 x$ Plan-Apochromat oil immersion objective (numerical aperture 1.40) or similar. Apply immersion oil and secure the dish in a circular stage insert such that the larvae face with their rostral aspect to the left.

2. Under bright-field or differential interference contrast illumination, select one of the mounted larvae for imaging. Adjust the focus with the focus knob such that the skin on the side of the fish closest to the coverslip is in focus, recognizable by the fingerprint-like pattern of periderm cells.

3. Once in focus, switch to epifluorescent illumination in the GFP channel. Locate the posterior lateral line by GFP expression along the horizontal myoseptum. Rings of fluorescent cells indicate neuromast mantle cells, and elongated strands of cells are interneuromast cells.

4. Beginning with the primlL1 neuromast, usually located just dorsal to the yolk, use the stage control joystick or other stage movement control to visually scan caudally along the horizontal myoseptum.

1. Follow the string of interneuromast cells until reaching the region between the primlL3 and primlL4 (L3 and L4) neuromasts (Figure 1A).

NOTE: Other regions can also be targeted, but this section of the trunk and tail tends to be closest to the cover glass and, therefore, most amenable to ablation.

2. If imaging several larvae, set the first stage position, then inactivate the multiple stage positions option by deselecting the appropriate check box.

3. Repeat steps 6.4.1-6.4.2. for each desired stage position (each larva).

5. After cell bodies in the L3-L4 region have been identified, switch to the acquisition mode.

1. Activate a GFP imaging track using the $488 \mathrm{~nm}$ or $491 \mathrm{~nm}$ laser.

2. Add a transmitted light (transmitted light photomultiplier tube or T-PMT) channel to the activated $488 \mathrm{~nm}$ laser track by activating the TPMT checkbox under the "Imaging Setup" dropdown menu.

3. Set the laser power to $6 \%$, pinhole size to 1 Airy unit equivalent, and digital gain to 750 for imaging ET20 larvae. Exact gain and laser power may vary for any given larva, depending upon how close to the cover slip they are mounted and the brightness of fluorescence. Adjust the gain such that cell bodies are saturated to capture otherwise dim projections and filipodia.

4. Adjust the parameters to a frame size of 1024 pixels $\times 1024$ pixels, digital zoom of $0.7(145.2 \mu \mathrm{m} \times 145.2 \mu \mathrm{m}$ image size $)$. Set averaging to 2 to improve image quality.

6. Check the z-stack box to bring up the z-position dropdown menu. While fast scanning, focus out until the interneuromast cells are just out of focus. Set the first slice, then focus through the sample until the interneuromast cells are once again out of focus. Set the last slice. Ensure that it encompass a z-stack of approximately $25 \mu \mathrm{m}$. Set the imaging interval to $1 \mu \mathrm{m}$.

7. Start experiment to capture a pre-ablation z-stack (Figure 1B). If stage positions have been added, inactivate the positions option so that only the current position is imaged. Save the file once it is captured.

\section{Laser ablation of cell bodies}

1. Click on "Show all Tools". This option is located at the top of the acquisition interface.

2. Click on the dropdown menu for "Imaging Set Up". In "Imaging Set up", click on "Add a new track" (represented as "+").

3. In "Imaging Set Up", click on the dropdown menu for "Dye". Select DAPI as the dye.

4. Click on the dropdown menu for "Channels" and deselect all other tracks by unclicking their respective checkboxes. Ensure that only the DAPI track is selected.

5. In the DAPI track, click on 405 for the "Laser setting". Increase the laser power to $75 \%$. Unclick the DAPI channel to turn off the laser while scanning for candidate cell bodies for ablation.

6. With the body of an interneuromast cell centered in the field of view, zoom in the scanning frame to $20 x-22 x$. The frame position may need to be adjusted to keep the cell body in the center as the zoom is applied. Stop live scanning as soon as the cell body fills the field of view. NOTE: The cell body should occupy the entire field of view. At this zoom level, the GFP will bleach rapidly. Minimize laser exposure time by working quickly. Use zoom rather than selecting a region of interest to increase laser power distribution over a small area.

7. Check the $405 \mathrm{~nm}$ laser shutter box to activate the track. Adjust the laser power to $75 \%$. Set a timer for $45 \mathrm{~s}$. Activate continuous scanning and start the timer. Stop scanning immediately at $45 \mathrm{~s}$.

\section{Post-ablation imaging and time-lapse microscopy to study regeneration}

1. Click on the dropdown menu for "Channels". In channels, unclick the "DAPI" track to inactivate the ablation laser. Click on the dropdown menu for "Acquisition mode". 
2. In acquisition mode, click on "Zoom".

3. Decrease the zoom to 0.7 either by using the slider or by typing in "0.7."

1. To ensure successful cell ablation, increase the gain to 900 and fast scan the field of view. NOTE: No GFP should be visible within the targeted cell or cells. If fluorescence is still observed, return to steps 7.1-7.3.

2. Using the same or similar settings as those described for pre-ablation imaging, capture and save a post-ablation image (Figure 1C) If the image reveals remnants of fluorescent cells or additional cell bodies that needs to be removed, repeat the laser ablation steps to create a visible gap in the string of interneuromast cells.

3. Inspect the T-PMT channel image to further confirm cellular damage. Damaged cells will have a granular appearance, and frequently the nuclei will swell or become irregular in shape (Figure 2).

4. After capturing a pre-ablation image, ablating the cells as necessary, and capturing a post-ablation image for each individual stage position, set up the time-lapse microscopy by activating both stage position and time options for image capture. Set the time parameters to $24 \mathrm{~h}$ or another desired endpoint and 15 min intervals. Start experiment to acquire images and save the resulting file when complete (on the following day).

5. If larvae are to be further studied or raised, carefully remove them from the agarose using fine forceps, then transfer into E3 medium without tricaine for recovery. If they are not to be used further, euthanize according to the approved animal protocol (rapid and sustained immersion in an ice bath is a common method) and dispose of them as required by the institution.

\section{Image analysis}

1. Open a post-ablation z-stack file in the preferred image processing software (Table of Materials). Split the channels such that each channel is in a separate viewing pane.

2. In the GFP channel window, use the line tool to draw a straight line between the tips of the remaining INMCs. This may be aided by scrolling up and down through the z-stack or by performing a maximum-intensity projection prior to drawing the line.

3. Use the "Measure" tool to obtain the distance between INMC ends in the $x$-and y-planes.

4. Scroll through the z-slices in the original z-stack to determine the distance between INMCs in the z-plane.

5. Calculate the total distance between INMC ends using the Pythagorean theorem $\left(a^{2}+b^{2}=c^{2}\right)$. The hypotenuse is the total distance (or gap size).

6. Enter the resulting distance measurement into a spreadsheet application for data analysis.

7. Review the time-lapse microscopy files collected in step 8.2-8.3 using either the integrated imaging software on the confocal system or freely available image analysis software. Identify the timepoint at which the gap between interneuromast cells was filled in by cell projections. This can be aided by examination of multiple z-slices to confirm closure of the gap in all dimensions.

8. To achieve the most accurate measurement of time to gap closure, use the post-ablation image time stamp (visible in Finder or File Explorer) as the zero time point; measuring time from the beginning of the time-lapse image stack may result in underestimating time to closure, depending upon how much time elapsed while ablating additional stage positions, etc.

9. Derive the rate of closure by simply dividing the original gap size $(\mu \mathrm{m})$ by the hours or minutes required for complete gap closure.

\section{Representative Results}

In order to ablate INMCs and record regeneration, screened GFP-expressing zebrafish larvae of the ET20 transgenic line were mounted at 2or 3-days post-fertilization for ablation as described in step 5.4. Several fish can be mounted simultaneously so that multiple time lapses can be captured in a single experiment. The region of the lateral line located between primlL3 and primlL4 neuromasts was identified, and pre-ablation images were captured as described in steps 6.5-6.7 (Figure 1A,B).

Three cell bodies were targeted for laser ablation to create a gap in the INMC string of approximately $40 \mu \mathrm{m}$. Post-ablation scanning with high gain and subsequent imaging confirmed that no cell bodies remained in the ablated region, leaving a gap between elongated projections of the adjacent INMCs (Figure 1C). Cell death was further demonstrated by examining the T-PMT channel after ablation. Damaged and dying cells were marked by swollen and irregularly shaped nuclei as well as a granular appearance (Figure 2, outlined). In some cases, time-lapse imaging also revealed the recruitment of large amoeboid cells that were likely macrophages (Figure 2, asterisk). Dark areas surrounding the ablated INMCs indicated photobleaching in overlying periderm cells. These cells did not appear to be damaged or destroyed by laser irradiation in these experiments; rather, their fluorescence was temporarily reduced. Time-lapse microscopy reveals that these cells normally recover after ablation and do not change shape or otherwise appear damaged (unpublished results, Volpe et al.).

To support the specificity of INMC destruction, ablations were performed in double-transgenic ET20 and Tg(neuroD:tdTomato) larvae in which the lateral line nerve (which runs just microns below the interneuromast cells) is labeled with red fluorescent protein. Ablations of several cells that created sizeable gaps in the INMC string had little or no effect on the lateral line nerve based on red fluorescence (Figure 3). Flexibility of the technique for ablating superficially localized cells was demonstrated by selective destruction of individual sensory hair cells within neuromasts of the lateral line. Using essentially the same protocol detailed above (sections 7 and 8 ), single hair cells in transgenic $T g$ (myo6b:ßactin-GFP) larvae were destroyed without apparent damage to adjacent cells (Figure 4). The ablated hair cells did not recover fluorescence after time-lapse microscopy, and their nuclei were noticeably granular and misshapen after laser exposure (Figure 4B). Similar to INMC ablations, apparent macrophages were frequently recruited to the laser exposure site (unpublished results, Volpe et al.).

Following laser ablation and post-ablation image capture, gap size was measured using freely available image analysis software. The measure tool demonstrated gap sizes ranging from just a few microns up to 100 microns, depending upon the width of individual INMCs and how many cells were chosen for ablation (Figure 5). Timelapse microscopy was employed to record INMC behaviors during regeneration. In 50 trials, 15 gaps (30\%) were closed by regeneration within a $24 \mathrm{~h}$ period. In most cases, INMCs that were able to recover did so within the first several hours of imaging. Recovery was defined as the timepoint at which projections from neighboring cells came into contact, which occurred $16 \mathrm{~h}$ after ablation for a gap of approximately $40 \mu \mathrm{m}$ (Movie 1). Z-stacks were carefully examined to ensure that cell-cell contact occurred within a 
single z-plane, avoiding possible artifacts due to z-projections. Logistic regression analysis revealed that the probability of gap closure correlated with gap size $(p=0.0453)$, with smaller gaps being more likely to heal. A gap of $55.5 \mu \mathrm{m}$ yielded a closure probability of $50 \%$ (Figure 5 ).

In $70 \%$ of cases, INMCs were unable to completely close the gap created by ablation. However, even in these cases we were able to monitor the formation of long projections from neighboring INMCs, which resemble in some respects extending neuronal growth cones (Movie 2). Thus, these experiments can also provide insight into the behaviors of INMCs after damage.
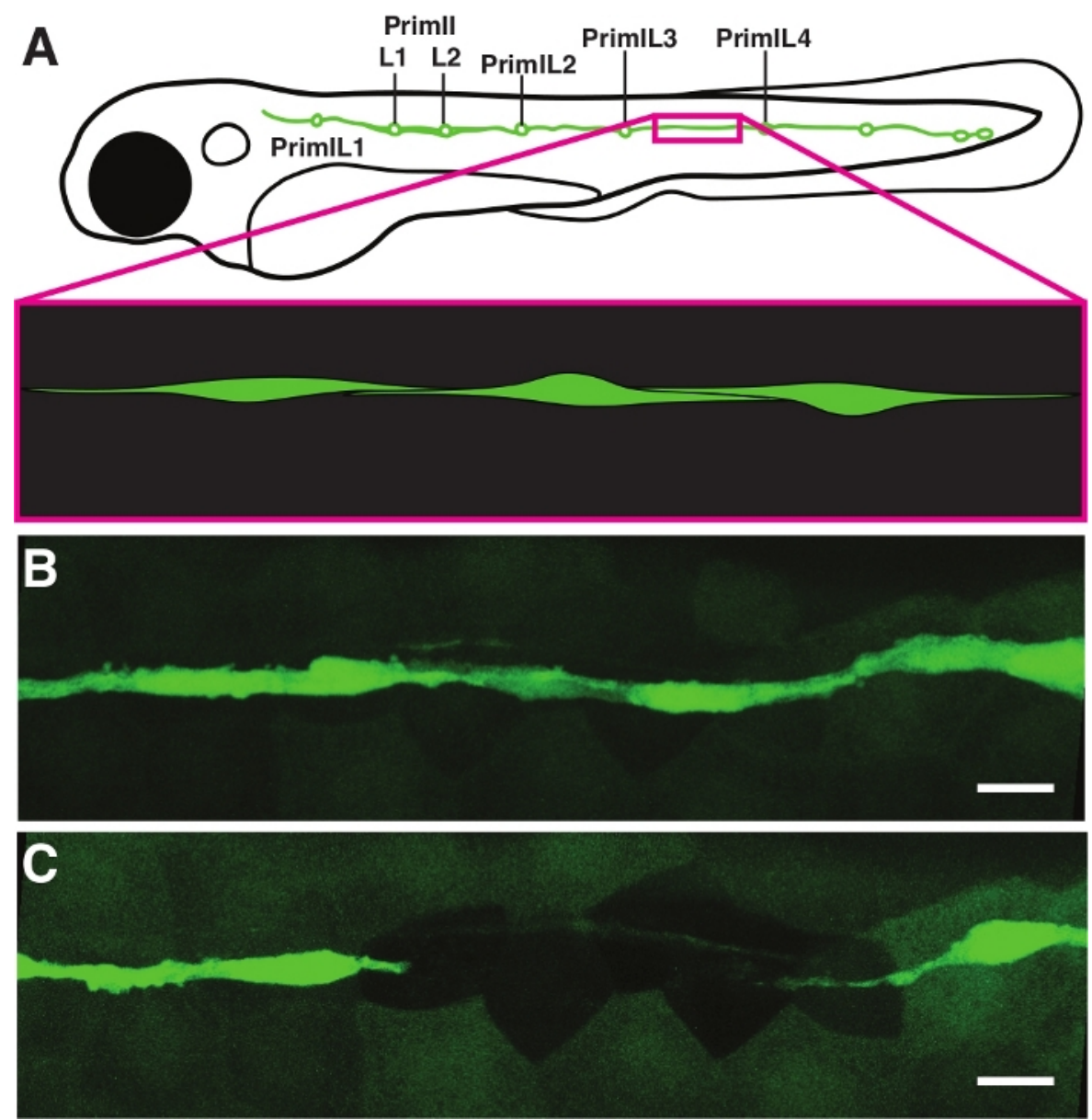

Figure 1: Selective ablation of interneuromast cells by laser irradiation. (A) Interneuromast cells between neuromasts primIL3 and primIL4 were selected for ablation. These cells display a characteristic spindle shape with elongated projections overlapping adjacent cell bodies. (B) Pre-ablation imaging identified cell bodies that could be ablated to produce a gap. (C) Post-ablation imaging confirms successful ablation as indicated by the absence of cell bodies in the gap region. Scale bars $=10 \mu \mathrm{m}$. Please click here to view a larger version of this figure. 

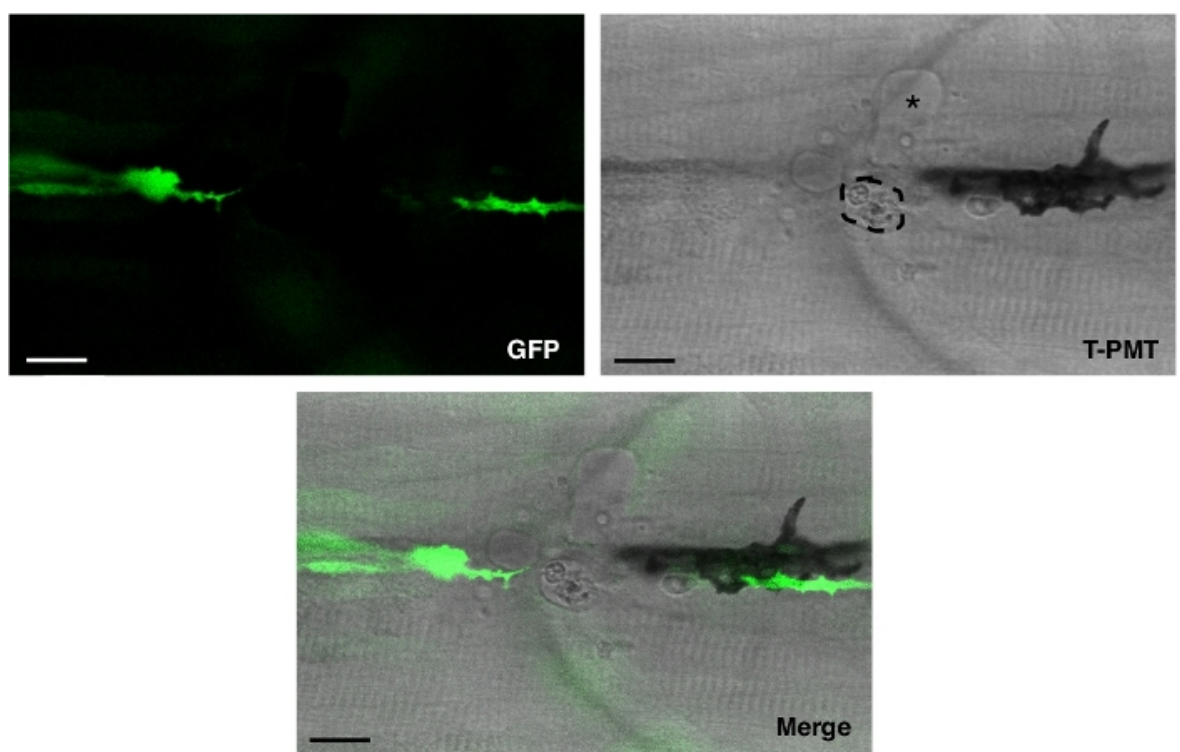

Figure 2: Post-ablation imaging demonstrates cell death in response to laser exposure in GFP-labeled interneuromast cells. Transmitted light photomultiplier imaging (T-PMT) indicates the presence of a necrotic cell with a granular appearance (encircled) as well as the recruitment of irregularly shaped cells that are likely macrophages $\left(^{*}\right)$. Merging of GFP and T-PMT channels confirms that cell death and macrophage activity occurred at the site of ablation. Scale bars $=10 \mu \mathrm{m}$. Please click here to view a larger version of this figure.
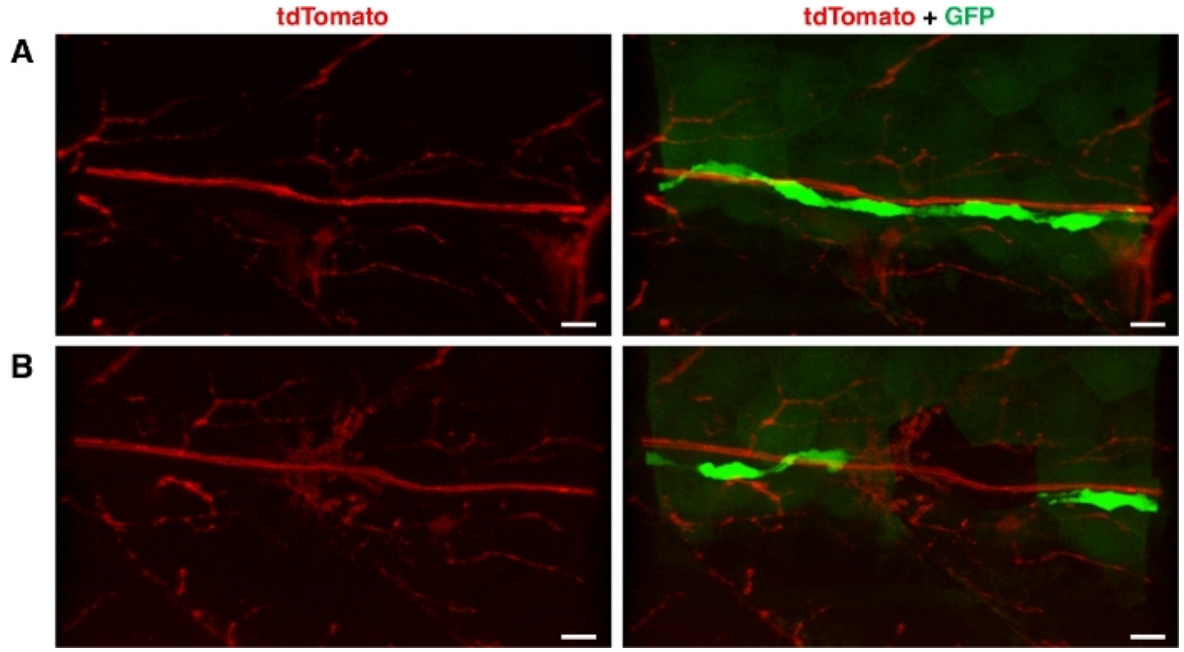

Figure 3: Specificity of interneuromast ablation. (A) Pre-ablation GFP-labeled interneuromast cells (green) and tdTomato-labeled lateral line nerve (red) in a double-transgenic $\operatorname{Tg}(E T 20 ;$ NeuroD:tdTomato) larva. Cell bodies in close proximity to the lateral line nerve were targeted for ablation. (B) Post-ablation imaging demonstrates ablation of targeted cell bodies with an intact lateral line nerve. Scale bars $=10 \mu \mathrm{m}$. Please click here to view a larger version of this figure. 


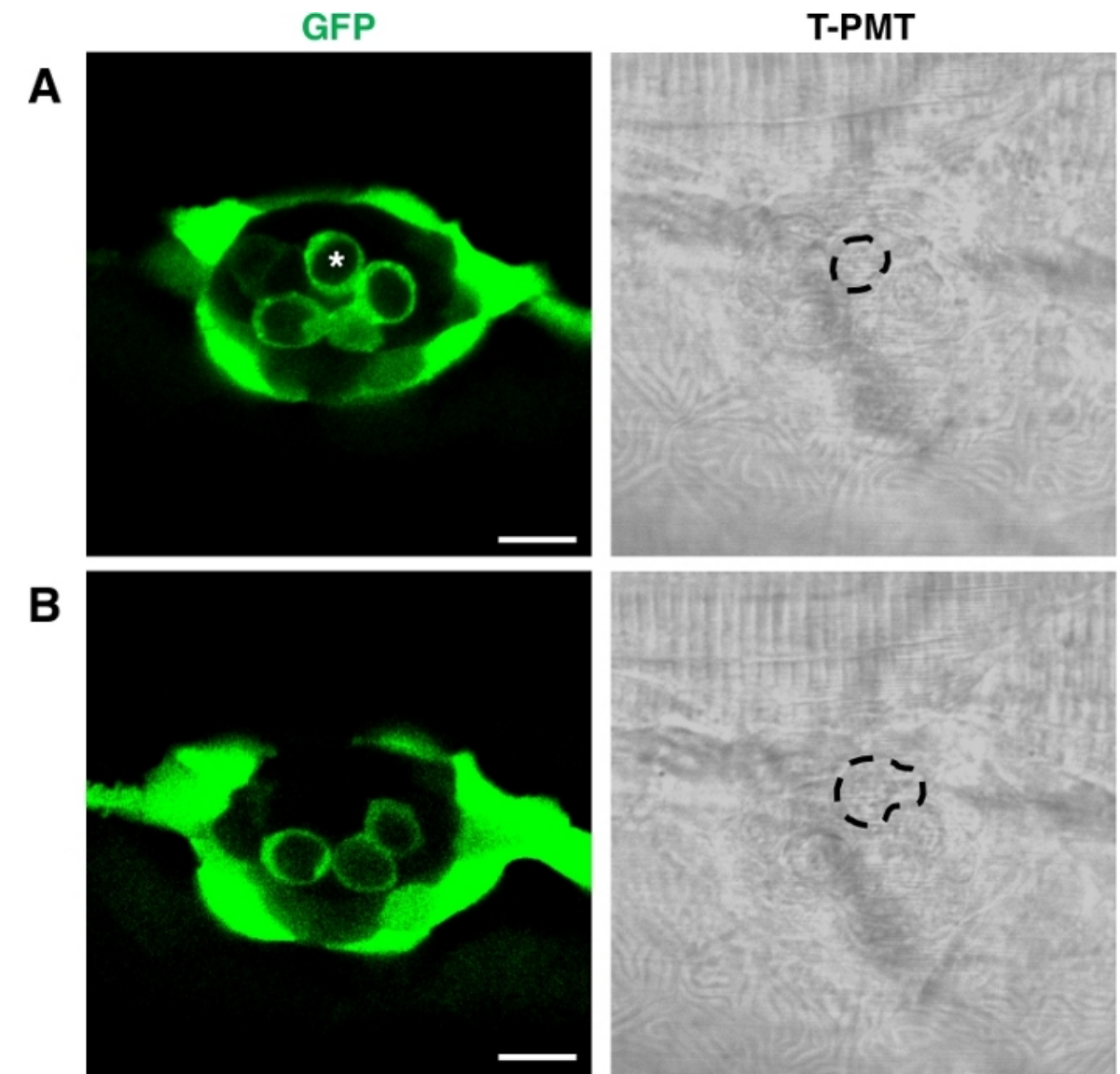

Figure 4: Ablation of sensory hair cells using confocal microscopy. (A) Pre-ablation imaging identified a GFP labeled sensory hair cell ${ }^{*}$ ) targeted for ablation in double-transgenic Tg(ET20; myo6b:ßactin-GFP) zebrafish. Transmitted light photomultiplier tube (T-PMT) imaging discloses normal hair cell morphology, with a round cross-section. (B) Post-ablation imaging confirms successful ablation of the targeted hair cell. A T-PMT image indicates irregularity in hair cell shape and increased granularity after laser exposure, suggesting cell death rather than photobleaching. Scale bars $=10 \mu \mathrm{m}$. Please click here to view a larger version of this figure. 


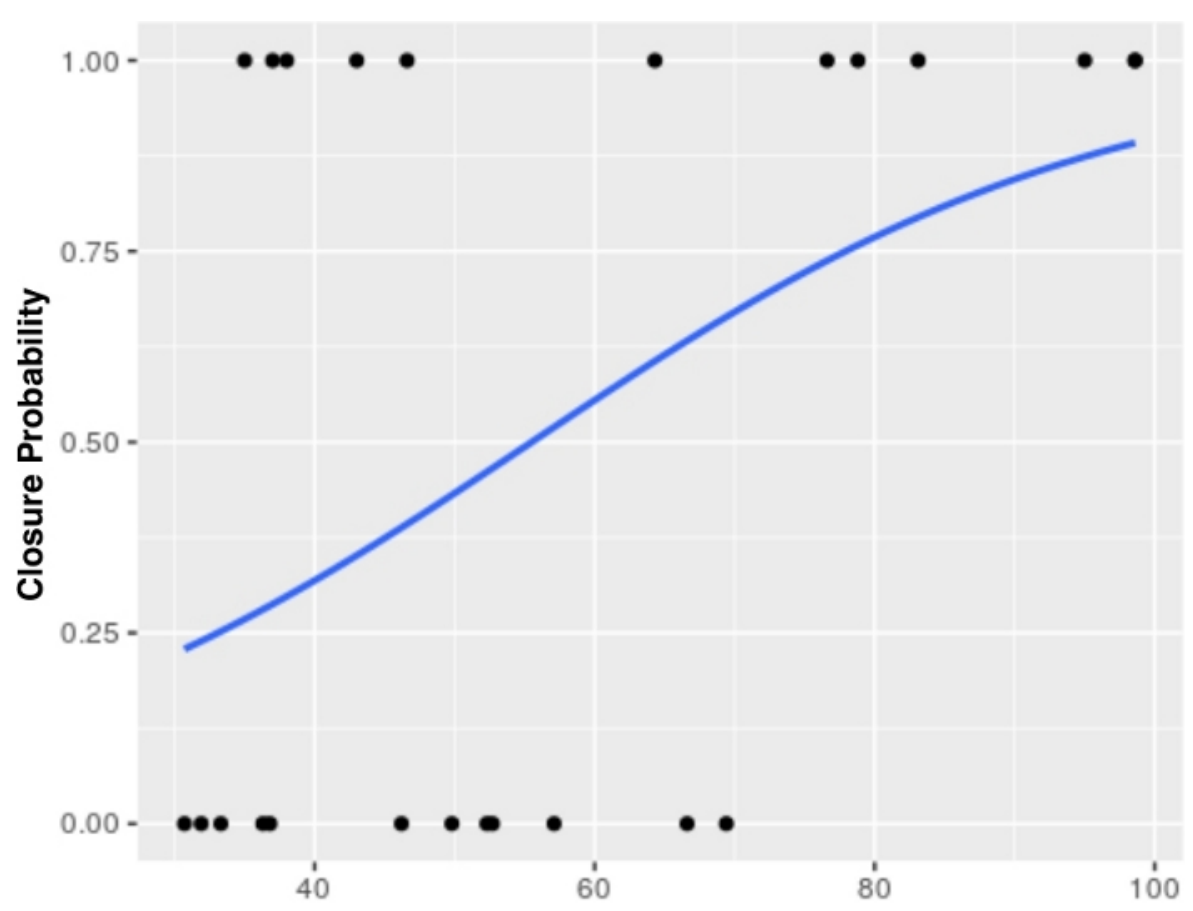

Gap Width $(\mu \mathrm{m})$

Figure 5: Logistic regression models the probability of gap closure as a function of gap width (in $\mu$ m). A score of 0 represents complete gap closure, whereas a score of 1 represents incomplete gap closure. Results indicate that the effect of gap width on the ability of interneuromast cells to close respective gaps is statistically significant ( $p=0.0453, n=24$ total; $n=12$ closed gaps, $n=12$ incompletely closed gaps). Please click here to view a larger version of this figure.

Movie 1: Time-lapse microscopy demonstrating gap closure $(\mathbf{4 0} \boldsymbol{\mu m})$ after $\mathbf{1 6} \mathbf{h}$. Images were captured every 15 min, and a maximumintensity z-projection was made for all timepoints. Please click here to download this video.

Movie 2: Time-lapse microscopy of an INMC gap that did not close. The elongated and branching projections of the remaining INMCs are shown. Please click here to download this video.

\section{Discussion}

The protocol describes a versatile method for laser cell ablation that can be performed on any confocal microscope equipped with a nearultraviolet laser ( $405 \mathrm{~nm}$ wavelength). This protocol addresses limitations of previously employed methods such as electroablation (which causes more widespread damage ${ }^{11}$ ) and pulsed UV-laser ablation (which requires additional specialized equipment ${ }^{12}$ ). Pre- and post-ablation confocal imaging provide rapid feedback regarding success of the experiment. Subsequent time-lapse microscopy offers a simple assay for regeneration in a cell type critical for sensory system development.

It is of vital importance to prescreen for transgenic zebrafish that express GFP strongly in neuromasts and INMCs. Larvae with bright fluorescence and approximately even spacing of the priml-derived neuromasts are ideal candidates for laser ablation. Even in these fish, there are occasionally dimmer INMCs that may at first escape detection. These cells can remain behind after laser ablation, preventing the formation of a true gap between INMCs. The digital gain should be adjusted to reveal these dimmer cells during pre-ablation imaging such that they can also be targeted with the $405 \mathrm{~nm}$ laser (step 8.2).

Similarly, laser targeting sometimes will not entirely destroy a cell; rather, it will bleach the fluorescence, resulting in a failure to create a real gap. These cells will generally increase in fluorescence during timelapse microscopy. Gain adjustment in the post-ablation imaging step along with T-PMT imaging (Figure 2) help ensure that all cells targeted have been effectively removed. It frequently requires two or three individual cell ablations to produce a gap in the INMC string. Cell death can be detected by blebbing or a granular appearance in the targeted cells; although, this may require a brief waiting period after laser targeting (and in some cases, apparent apoptotic or necrotic figures are observed shortly thereafter).

A limitation of this procedure is that it may require multiple experimental trials before laser conditions are optimized and INMC regeneration is successful. The laser power and total dwell time of the laser may each require slight adjustment for different samples. It has been found that excessive laser application can impair the ability of the lateral line to repair itself. This includes both 1) overexposure of individual cells to laser irradiation, presumably causing additional damage to surrounding tissues, as well as 2) the ablation of too many cells in the string, leading to a larger gap. Users must achieve a balance between sufficiently irradiating cells to ensure their destruction and not overexpose the cells, which can slow or prevent regeneration. With the appropriate settings, it has been found that the INMCs can be removed without visible damage to the posterior lateral line nerve, indicating that collateral damage is minimized with this protocol unlike electroablation ${ }^{11}$. In no cases were new 
neuromasts forming in the gap observed, presumably because the lateral line nerve remains intact and underlying glial cells remain and inhibit the proliferation of INMCs $s^{6,7,8,11}$.

It is demonstrated that this method of confocal laser ablation may be applied to other cell types as well, particularly in those superficially located within the animal including sensory hair cells (Figure 4). A similar protocol may be used to ablate skin cells to examine wound repair or neurons for axonal regeneration studies, as previously described ${ }^{13}$. It is anticipated that this technique will become a useful addition to the experimental repertoire of laboratories that possess a confocal microscope but no other specialized equipment for laser ablation.

\section{Disclosures}

The authors have nothing to disclose.

\section{Acknowledgments}

This work was funded by NIH R15 grant 1R15DC015352-01A1 and Pace University internal funding sources. Fish lines were courtesy of Vladimir Korzh ${ }^{14}$, Katie Kindt ${ }^{15,16}$, and the laboratory of A. James Hudspeth. We would like to thank A. James Hudspeth and members of his group for feedback on these experiments, and colleagues at Pace University for their support. Logistic regression analysis in RStudio was aided in particular by our colleague Matthew Aiello-Lammens.

\section{References}

1. Denans, N., Baek, S., Piotrowski, T. Comparing Sensory Organs to Define the Path for Hair Cell Regeneration. Annual Review Cell \& Developmental Biology. 35, 567-589 (2019).

2. Ghysen, A., Dambly-Chaudiere, C. The lateral line microcosmos. Genes \& Development. 21 (17), $2118-2130$ (2007).

3. Lush, M. E., Piotrowski, T. Sensory hair cell regeneration in the zebrafish lateral line. Dev Dyn. 243 (10), 1187-1202 (2014).

4. Ledent, V. Postembryonic development of the posterior lateral line in zebrafish. Development. 129 (3), 597-604 (2002).

5. Nunez, V. A. et al. Postembryonic development of the posterior lateral line in the zebrafish. Evolution and Development. 11 (4), 391-404 (2009).

6. Grant, K. A., Raible, D. W., Piotrowski, T. Regulation of latent sensory hair cell precursors by glia in the zebrafish lateral line. Neuron. $\mathbf{4 5}$ (1), 69-80 (2005).

7. Lopez-Schier, H., Hudspeth, A. J. Supernumerary neuromasts in the posterior lateral line of zebrafish lacking peripheral glia. Proceedings of the National Academy of Sciences U. S. A. 102 (5), 1496-1501 (2005).

8. Lush, M. E., Piotrowski, T. ErbB expressing Schwann cells control lateral line progenitor cells via non-cell-autonomous regulation of Wnt/betacatenin. eLife. 3, e01832 (2014).

9. Hernandez, P. P. et al. Sublethal concentrations of waterborne copper induce cellular stress and cell death in zebrafish embryos and larvae. Biological Research. 44 (1), 7-15 (2011).

10. Moya-Diaz, J. et al. Electroablation: a method for neurectomy and localized tissue injury. BMC Developmental Biology. 14, 7 (2014).

11. Sanchez, M., Ceci, M. L., Gutierrez, D., Anguita-Salinas, C., Allende, M. L. Mechanosensory organ regeneration in zebrafish depends on a population of multipotent progenitor cells kept latent by Schwann cells. BMC Biology. 14 27, (2016).

12. Viader-Llargues, O., Lupperger, V., Pola-Morell, L., Marr, C., Lopez-Schier, H. Live cell-lineage tracing and machine learning reveal patterns of organ regeneration. eLife. 7, (2018).

13. Morsch, M. et al. Triggering Cell Stress and Death Using Conventional UV Laser Confocal Microscopy. Journal of Visualized Experiments. (120), e54983 (2017).

14. Parinov, S., Kondrichin, I., Korzh, V., Emelyanov, A. Tol2 transposon-mediated enhancer trap to identify developmentally regulated zebrafish genes in vivo. Developmental Dynamics. 231 (2), 449-459 (2004).

15. Kindt, K. S., Finch, G., Nicolson, T. Kinocilia mediate mechanosensitivity in developing zebrafish hair cells. Developmental Cell. 23 (2), 329-341 (2012).

16. Toro, C. et al. Dopamine Modulates the Activity of Sensory Hair Cells. Journal of Neurosciences. 35 (50), 16494-16503 (2015).

17. Dalle Nogare, D., Chitnis, A. B. A framework for understanding morphogenesis and migration of the zebrafish posterior Lateral Line primordium. Mechanisms of Development. 148, 69-78 (2017). 